\title{
BIBLIOGRAPHIE.
}

[1] Chr. Barther, Zeitschr. f. Unters. Nahrungs und Genussmittel, 34, 137, 1917 ,

[2] R. S. BREED. Zentr. f. Balot. A., II, 30, 337, 1911.

[3] R. E., Buchanan. Abstr. Baet., 2, 11, 1918.

[4] R. BURRI. Le Lait, 9, 1028, 1929.

[5] S. DuUzewskr. Polska Gazeta Lekarska, 8, nº 32, 1929.

[6] W. DonNer. Le Lait, 13, 595, 1933.

[7] W. Dorner et P. Demont. Le Lait, 9, 909, 1931.

[8] R. A. FISHER. Statistical Methods for Research Workers, table V A., 1930.

[9] J. HrRsch. Klin. Wochenschr., 12, 191, 1933.

[10] E. Löfrler et R. Rigler. Zentr. f. Bakt., A. I, 99, 1, 1926.

[11] M. M. MAson. Journ. of Bact., 29, 103, 1935.

[12] T. Matuszewskr. Medycyna Doswiadcz. i Spoleczna, 18, z. 5-6, 1934.

[13] K. L. Pesch et U. Stmment. Milchwirtsch. Forschungen., 8, 551, 1929.

[14] O. RAHN. Zentr. f. Batet., A. II, 32, 375, 1912.

[15] O. SkaR. Intern. Milchw. Kongr. Vorträge 2 Seltion, Deutsche Ausg., $58,1931$.

[16] R. H. Thoraton et E. G. Hastinas. Journ. of Dairy Science, 13, 221, 1930.

[17] T. Thunbera, C. Oppenhermer et L. Pinaussen. Die Methodite der Fermente, 1118, 1929.

[18] H. H. Walker, C. E. A. Winslow, E. Huntington et M. G. Mooney. Journal of Bact., 27, 303, 1934.

\section{UN COEFFICIENT DE PROPRETÉ DU LAIT}

\section{par le Dr ALBERT FOURNIER}

membre de la Ligue du Lait.

Dans la séance du Conseil d'administration de la «Ligue du Lait " réuni chez son secrétaire général, M. Ch. Grollet, le 28 mars 1935, j'ai proposé la détermination d'un coefficient d'un indice de propreté du lait, basé sur la détermination quantitative et qualitative de ses impuretés solides (1).

La propreté du lait est en effet une des qualités particulièrement souhaitée par la "Ligue du Lait". Les souillures n'apparaissant pas sont méconnues par le consommateur et le plus souvent sans importance pour le producteur vis-à-vis duquel, en général, la propreté est une notion peu familière. Seul l'industriel la connaît, mais reste impuissant en raison de l'indifférence générale. La " Ligue du Lait "n'ignore pas le beau rôle qu'elle peut jouer auprès du public en lui faisant connaître que le lait n'étant pas propre, elle s'est donné pour tâche d'encourager sa propreté. Une opinion publique pourrait être facilement créée dans ce sens, surtout par la voie de la Presse qui touche aujourd'hui à peu près toute la collectivité.

(1) Revue de Pathologie comparée. No 465, juin 1935, p, 723. 
Si les industriels du lait s'accordaient pour refuser aux producteurs un lait dont le coefficient de propreté serait inférieur à une certaine limite, si d'autre part ces derniers étaient encouragés à mieux faire, soit par les administrations communales, soit par des récompenses de ces dernières ou de la "Ligue du Lait ", nul doute que certaines précautions élémentaires seraient prises par eux.

Mais en dehors du sentiment de répulsion que peuvent éprouver les consommateurs qui savent, y a-t-il vraiment un intérêt, au point de vue hygiénique, au point de vue de la santé générale et de celle des enfants en particulier, y a-t-il vraiment un intérêt à la consommation d'un lait propre?

La réponse ne paraît pas douteuse. De quoi, en effet, sont composées les souillures ordinaires du lait, indépendamment de toute malveillance et simplement fonction de l'ignorance, des négligences, de l'impuissance du producteur et des intermédiaires, jusqu'au consommateur?

A la production, ce sont des souillures d'origine animale (poils, squames épidermiques, matières fécales, urine, etc.); d'origine végétale (litière, foin, paille, débris alimentaires végétaux provenant de l'intestin, des pâturages ou des étables) ; d'origine minérale (calcaire, sable, etc.). Enfin, des mélanges complexes issus du sol ou des poussières de l'air, avec accompagnement de tous les microbes saprophytes ou pathogènes répandus partout et principalement dans des lieux mal tenus. $Y$ ajouter encore les gaz ammoniacaux ou sulfurés originaires de fermentations diverses, gaz capables de se dissoudre dans le lait.

Un lait souillé est donc dangereux et par ses apports toxiques et par ses apports microbiens.

Existe-t-il pourtant un parallélisme entre la quantité des souillures autres que les microbes et la quantité, la nocivité de ces derniers ? Evidemment non, car quelques microbes de la tuberculose ou de la fièvre typhoïde seront assurément plus dangereux qu'un taux, même élevé, de certains débris, végétaux. C'est pourquoi à la quantité de souillures il importe de joindre l'étude de la nature de ces souillures. A la détermination pondérale, il faudra joindre, dans une première approximation, un examen microscopique; dans une seconde, si besoin était, un examen quantitatif et un examen qualitatif des germes vivants.

Déjà d'ailleurs la pesée des impuretés donnerait présomption du danger microbien, surtout si ladite pesée était complétée par un simple examen à la loupe.

L'étude plus complète du lait esi à souhaiter par un laboratoire qualitié, comme il est désirable que la "Ligue du Lait » en possède un (j'ai signalé à ce propos la création, par les industries pharmaceu- 
tiques, d'un laboratoire de contrôle de ces industries, mais indépendant d'elles) ou par des laboratoires annexés aux grandesindustries laitières. Ma proposition d'un coefficient de propreté a pour elle une simplicité et une utilité n'excluant pas d'ailleurs tout autre procédé ou méthode capable d'aider les hygiénistes du lait (hygiène des animaux, des vachers, des étables, instrumentation, etc.).

\section{RECHERCHE ET DOSAGE DES IMPURETÉS SOLIDES}

$1^{0}$ Centrifugation. - La centrifugation, notamment préconisée par Lотн, exige un appareil puissant et coûteux. Dans le lait centrifugé, on aperçoit au fond du tube des matières solides étrangères, visibles à l'ceil nu, puis par palliers successifs les divers composants du lait allant de bas en haut, de l'opacité maxima de ce liquide à une certaine opalinité. Enfin la crème surnage. Mais à l'œil, même armé d'une loupe, on ne peut discerner les souillures qui peuvent être retenues par ces plans successifs et notamment par la couche butyreuse. Comme le conseille Lотн, il faut done reprendre tout particulièrement la crème et lui faire subir un traitement susceptible de donner tous leurs effets à une ou plusieurs autres centrifugations.

$2^{\circ}$ Voir et doser les impuretés par sédimentation spontanée dans le lait rendu plus léger et transparent. - Est-il possible de détruire l'opacité du lait pour en permettre un examen visuel, tout en respectant les impuretés dans leur quantité et leur nature?

Pour résoudre ce problème, j'ai songé à la liqueur d'Adam capable, comme on sait, de rendre le lait translucide en détruisant son émulsion graisseuse et accessoirement ses pseudo-solutions colloïdales protidiques et minérales.

J'ai d'abord voulu m'assurer que la liqueur d'Adam, par ses proportions relatives, était bien la liqueur optima de clarification. Dans la somme $\mathrm{L}+\mathrm{A}=$ volume constant $(\mathrm{L}=$ lait, $\mathrm{A}=\mathrm{Adam})$ j'ai fait varier le rapport $\frac{L}{A}$ entre les limites $A=0, L=$ Volume maximum du lait $; \mathrm{L}=0, \mathrm{~A}=$ Volume maximum de la liqueur d'Adam.

J'ai trouvé ainsi que la composition de la liqueur, donnée par Adam, était bien la meilleure, comme il fallait d'ailleurs le prévoir.

On peut opérer dans l'appareil d'Adam, ce qui, en dehors des impuretés, permet au besoin de doser le beurre, la caséine et le lactose. Les matières solides forment un dépôt noirâtre au-dessus du robinet d'obturation. On les sépare en les faisant tomber dans un second appareil avec le moins possible de liquide surnageant. On les lave à deux ou trois reprises avec quelques centimètres cubes de liqueur d'Adam. Il suffit de les recevoir alors dans un verre de montre ou une capsule légère tarés, pour les peser après les avoir dosséchées. 
Je me suis assuré que la liqueur d'Adam, pendant le temps assez court des manipulations, ne dissolvait pas sensiblement certains des principes des matières étrangères et notamment les lipides, contrôle effectué sur poils, laine, coton, paille. Cet examen n'est d'ailleurs resté qu'approximatif, car je n'ai pas fait une étude systématique de cette question.

Les matières pour $10 \mathrm{~cm}^{3}$ du lait examiné provenant de mon laboratoire où il est utilisé à d'autres fins, ces matières, dis-je, paraissent insignifiantes et $j$ 'ai dû traiter un litre de lait pour obtenir 0 gr. 02 d'impuretés. Ceci montre la pureté relative du lait sur lequel j'ai opéré, filtré d'ailleurs et centrifugé avant pasteurisation. Ceci montre aussi tout l'intérêt qu'il y aurait à faire la détermination sur les laits avant leur traitement industriel, car si la filtration-centrifugation sépare les éléments étrangers les plus grossiers, elle n'a que peu d'effets sur la flore microbienne corrélative des souillures.

On dira : cette flore peut être à son tour annihilée par la pasteurisation. Sans doute, mais jusqu'à ce moment le lait n'en contient pas moins les corps microbiens et leurs toxines, dont la pullulation est d'autant plus active, à n'en pas douter, que les malpropretés du lait sont dès le début plus abondantes. Le traitement d'une maladie, même après guérison, n'en supprime pas toujours certaines lésions définitives. On dira encore : comment juger d'un lait par des souillures qui n'existent plus ? Il vaut mieux, dans ce cas, en évaluer les effets, par exemple par détermination de l'acidité. Juger des effets d'une cause inaccessible, c'est vouloir juger sans appel une maladie par ses symptômes, en déduire un traitement symptomatique, tandis que le seul traitement rationnel et efficace est le traitement étiologique. Si l'on ne démontre la malpropreté du lait que par ses effets dont le mécanisme de production reste d'ailleurs mystérieux au plus grand nombre, si l'on n'attaque pas le mal dans sa racine, non seulement en le dénonçant, mais en le mettant en quelque sorte sous les yeux de tous, on risque de perpétuer longtemps l'état de chose actuel.

L'impureté solide doit être requise et mise en évidence à l'entrée de l'usine, ou mieux encore chez le producteur quand son éducation sera faite, quitte à la rechercher à nouveau, cette impureté, jusque chez le détaillant.

Au surplus, la mesure de l'acidité avant coagulation, bien entendu, le lait perdant son nom dans cet état, ne prendrait toute sa valeur que par le dosage des acides totaux de fermentation, libres ou salifiés, et principalement de l'acide lactique, les autres étant pondéralement négligeables. Alors on pourrait joindre cette acidité lactique au coefficient de propreté, sous le nom de coefficient d'altération du lait. 
La liqueur d'Adam permet de juger immédiatement de la pureté relative d'un lait. Si, comme dans le cas précédent, les $10 \mathrm{~cm}^{3}$ de lait traité montrent l'insuffisance de ce volume, on le multipliera par deux, par trois, d'après l'importance visuelle du sédiment, et l'on pourra alors opérer dans une simple ampoule à robinet de capacité convenable, ou réunir les essais de plusieurs ampoules d'Adam.

$3^{\circ}$ Emploi de la filtration. - Divers appareils existent déjà : bouteille de Gerber, creuset de Gooch. J'ai employé le suivant, composé essentiellement d'un tube $T$ à filtration pouvant être fixé par bouchage émeri sur le goulot d'un vase cylindrique $\mathrm{V}$, ce goulot s'engageant dans le tube, la fermeture émeri lui étant donc extérieure. On verse un volume déterminé de lait, soit 1 litre, dans le vase V. Le lait traverse deux couches successives filtrantes formées : $1^{0}$ à la partie supérieure d'un feutrage d'amiante légèrement tassée; $2^{\circ}$ d'un feutrage de même nature mais beaucoup plus fin $\mathrm{F} ; 3^{\circ}$ enfin d'une couche de coton tassée modérément et dont aucune effiloche ne doit pénétrer dans le tube final. Ces différentes couches filtrantes sont successivement comprimées, ou mieux tassées, par un mandrin cylindrique à base plane s'ajustant exactement dans le tube $\mathrm{T}$ à la manière d'un piston dans son eylindre. La filtration peut être aidée au moyen du vide,

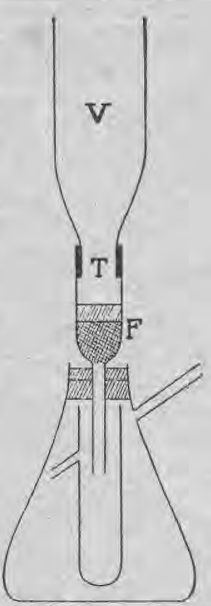
ainsi qu'on le voit sur la figure, mais ce vide doit être modéré et surveillé. Il est indispensable en tout cas que jusqu'à la fin de l'opération il y ait toujours du lait dans le tube $T$ pour éviter les à-coups d'aspiration. Finalement on aspire sans brutalité le volume résiduel final. On termine en lavant le filtre d'abord avec de l'eau pure, puis de l'eau ammoniacale, enfin de l'alcool et de l'éther. Il suffit alors d'isoler le tube $\mathbf{T}$, de le peser et, par différence, on aura les impuretés solides après dessiccation, si l'on a pris soin de tarer le tube $T$, et le filtre, dans les mêmes conditions de dessiccation. Naturellement la matière filtrante a été au préalable dégraissée et, de plus, l'amiante lavée par l'eau acidulée $\mathrm{HCl}$, l'eau pure, et ensuite incinérée au moufle pour en détruire les matières organiques. Il est encore plus pratique de peser la matière filtrante avant et après filtration, entre deux verres de montre. Après l'avoir fait tomber sur le verre de montre, en la saisissant au besoin avec une pince, on nettoie, s'il est nécessaire, le tube avec la pince munie d'un peu de coton sec dont on connait le poids et que l'on joint à la matière filtrante. 


\section{Comment concevoir un coefficient de propreté du lait ?}

Soit $\mathrm{p}$ le poids des impuretés solides, poids rapporté à un litre de lait. Définirons-nous le coefficient par le rapport $p$ au volume? Si la pesée donne, par exemple, 0,05 d'impuretés, dira-t-on que le coefficient d'impureté sera de 0,05 ? Mais, supposons ce lait étendu d'une certaine quantité d'eau. Pour fixer les idées, soit un quart de son volume. Le litre deviendra done 1.250 et les 0,05 d'impuretés seront dilués dans ce volume, en sorte que le coefficient réel 0,05 tomberait à $\frac{0.05 \times 1.000}{1.250}=0,04$, coefficient apparent masquant une seconde impureté, celle de l'eau, ajoutée aux premières.

Dans l'établissement du coefficient, il semble donc qu'il faille tenir eompte de la concentration moléculaire. Un moyen pour l'apprécier est l'évaluation du point cryoscopique, lequel a de plus l'avantage de fournir une donnée des plus intéressantes et même des plus précises parmi les données physiques, sur le mouillage du lait, qu'il soit accidentel ou voulu.

Le point cryoscopique ne varie qu'entre des limites très voisines pour le lait pur de chaque espèce animale. La concentration moléculaire de ce liquide, en effet, reste à peu près constante, quelles que soient les proportions relatives de ses composants, et à ce point de vue comme à beaucoup d'autres, il peut être comparé au sang.

Par conséquent, le coefficient de propreté du lait prendra plus de valeur si le rapport des impuretés solides à un certain volume de lait, le litre par exemple, est corrigé d'un mouillage possible décidé et mesuré par l'abaissement du point de congélation.

L'addition d'eau de l'exemple donné plus haut correspond à $\Delta=-0,44$. Les tables de correspondance entre les $\Delta$ et les dilutions aqueuses nous montreront précisément qu'un litre de lait examiné a été étendu à $1.250 \mathrm{~cm}^{3}$. Pour un litre de ce lait nous avons trouvé $\mathrm{p}^{\prime}=0,04$, coefficient ajparent. La correction nous fournit le coefficient vrai $\mathrm{p}=0,05$.

Pour établir notre rapport, nous aurions pu choisir une autre constante du lait au lieu du nombre $\Delta$. Mais, outre que ce dernier est assez facile à déterminer, il est de ceux dont les oscillations sont les plus faibles.

Parmi les autres, on peut songer aux composants dont la constance relative s'accompagne d'une fonction physiologique importante : tels le calcium et le phosphore, principalement sous leurs formes organiques. Le phosphore dans le lait de femme, on le sait, n'existe que sous cette dernière forme. Il préside, à n'en pas douter, à l'élaboration et à la construction de la charpente osseuse et de l'appareil nerveux du nourrisson. Dans le lait de vache, cette forme chimique n'est pas exclusive, mais, du moins, peut-on songer qu'elle 
devient physiologique dans l'allaitement de l'enfant, l'organisme de ce dernier faisant un choix dans l'aliment lacté animal dont il doit se contenter. Les lécithines existent dans les sécrétions mammaires des animaux comme dans celles de la femme. Leurs quantités oscillent entre des limites voisines, ce qui permettrait, mais avec moins de rigueur que le $\Delta$, d'évaluer le mouillage, car enrichir un lait en lécithine pour masquer une addition hydrique n'est pas à supposer, étant donné la difficulté d'incorporation, sans compter le prix élevé de la substance phosphorée.

Mais conviendrait-il alors de doser les phosphores lipoïdiques? S'il y a un intérêt évident à ce dosage, trop négligé de nos pédiâtres, pour évaluer la valeur nutritive et physiologique d'un lait destiné à l'enfance, il disparaît quand il s'agit de notre coefficient, devant la supériorité, à ce dernier point de vue, du $\Delta$.

Après le phosphore lipoïdien apparaissent dans le même ordre d'idée physiologique, le ealeium, l'acide citrique spéeifique de la sécrétion lactée, et d'autres encore.

Le Ca organique et la somme P + Ca organique pourrait également, comme le $\mathrm{P}$ organique, offrir le double intérêt de l'établissement d'un coefficient physiologique ajouté au coefficient de propreté en tenant compte de la richesse des éléments dosés en vue d'une correction de volume. En réalité, dans la pratique, la recherche du $\Delta$ luimême est le plus souvent inutile dans l'établissement d'un coefficient de propreté, car une addition d'eau comme celle de l'exemple cité plus haut ne se rencontre guère. Les dilutions accidentelles ou volontaires sont d'un ordre tel qu'elles n'apportent qu'une variation insignifiante à la valeur de $\mathrm{p}$ qui, d'autre part, ne pouvant pas être apprécié avec rigueur et n'étant qu'un indice de propreté, n'exige nullement en pratique la correction du mouillage.

\section{RÉSUMÉ ET CONCLUSIONS}

Pour apprécier la pureté du lait, nous avons à notre disposition l'analyse physico-chimique et l'analyse bactériologique. Cette dernière est corrélative de sa propreté et il existe incontestablement une relation entre la propreté, ou plutôt la malpropreté du lait, dans l'ordre pondéral et qualitatif et la qualité et la quantité des microbes, sans y voir cependant une question de proportionnalité.

La qualité hygiénique du lait est fonction évidemment de sa constitution bio-physico-chimique, mais en supposant qu'il représente la sécrétion lactée, exempte d'apports étrangers, autres que ceux ayant pour origine ses manipulations, à partir du moment où il est extrait de la mamelle, sa qualité hygiénique dépendra done des soins dont il sera l'objet depuis la traite jusqu'à sa eonsommation, et ces soins sont avant tout des soins de propreté et de réfrigération. 
L'apport microbien, nous le savoris, est fonction de la malpropreté. Déterminer l'un et l'autre par leur quantité et leur nature est souhaitable, car ils se complètent.

L'acidité (1) est un témoin de l'activité microbienne au début de l'altération du lait jusqu'à sa coagulation. Apprécier l'apport microbien par le dosage de l'acidité libre ne me paraît pas suffisant, car l'acidité est trop variable avec un grand nombre de facteurs : température, temps, neutralisation volontaire par les sels alealins $\left(\mathrm{CO}^{3} \mathrm{HNa}, \mathrm{CO}^{3} \mathrm{Ca}\right.$, etc...).

Le dosage du lactose et surtout le dosage de l'acide lactique total serait préférable, et encore mieux la numération des germes et la recherehe des variétés pathogènes.

Mais cette dernière étude exige la compétence d'un bactériologiste. Au contraire, la recherche, la pesée et même l'étude microscopique des impuretés solides et la détermination du $\Delta$, peuvent être le fait d'une simple "laborantine " exercée à ce travail, lequel pourrait être quotidien et répété autant de fois qu'il le faudrait dans le courant de la journée, en raison de sa rapidité et de sa facilité.

Ceci, bien entendu, je le répète, n'exclurait pas une étude plus complète dans certains cas particuliers.

En conclusion, je propose un coefficient de propreté défini de la manière suivante : Désignons par 100 le coefficient de propreté absolue, c'est-à-dire l'absence d'impuretés coīncidant avee un $\Delta$ normal (lait tel qu'il est sécrété par la mamelle). 100 - p représente la propreté relative qu'on pourra done évaluer sur l'échelle de 100 (pour $\mathrm{p}=0$, lait propre à $100 \%$ ) jusqu'à 0 (lait sale à $100 \%$ ).

Comme les impuretés sont représentées habituellement par des centigrammes, et qu'au point de vue "psychologique " il est bon d'user des pourcentages dont le public subit aujourd'hui particulièrement l'influence, nous affecterons du facteur 100 le poids des impuretés. Le coefficient de propreté s'exprimera par la relation suivante :

$$
100-100 \mathrm{p}=\mathrm{K}
$$

Il égalera 100 quand la propreté sera parfaite $(\mathrm{p}=0$, cas idéal) et prendra successivement les valeurs suivantes, diminutives au fur et à mesure de l'aggravation des souillures :

$$
\begin{aligned}
& 100-100 \times 0,00=100, \text { propreté à } 100 \% \\
& 100-100 \times 0,01=99, \text { propreté à } 99 \% \\
& 100-100 \times 0,05=95, \text { propreté à } 95 \%
\end{aligned}
$$

(1) Principalement fonction des ferments lactiques. Je ne considère ici que le lait exempt de certains traitements, comme la chaleur, qui, tuant les ferments lactiques, permet alors l'évolution de certains autres microbes. Les fermentations lactiques intermédiaires peuvent alors céder immédiatement la place à d'autres altérations portant par exemple sur les protides (peptonisation, aminogénie, etc.). On peut citer le cas du subtilis dont les spores résistent aux hautes températures. 


$$
\begin{aligned}
& 100-100 \times 0,10=90, \text { propreté à } 90 \% \\
& 100-100 \times 0,50=50, \text { propreté à } 50 \% \\
& 100-100 \times 1,00=00, \text { propreté nulle }
\end{aligned}
$$

Nous supposons qu'un gramme de malpropreté par litre doit être la limite supérieure qu'on puisse observer, sauf cas exceptionnels.

Si $\Delta$ nous indique un mouillage du lait $=\mathrm{A}$ (évalué en centimètres cubes) pour 1000 de lait, ce litre de lait composé de A d'eau + L de lait non mouillé, contient done $\mathrm{p}$ d'impuretés relatives par conséquent à $\mathrm{L}=(1000-\mathrm{A})$ de lait non mouillé. $\mathrm{p}$ corrigé du mouillage devient done $\mathrm{p}^{\prime}$ :

$$
\frac{p^{\prime}}{1000}=\frac{p}{1000-A} \text { d'où } p^{\prime}=\frac{1000 p}{1000-A}
$$

Et pour le coefficient de propreté réel :

$$
100-100 \times \frac{1000 \mathrm{p}}{1000-\mathrm{A}}=\mathrm{K}
$$

Si par exemple on a trouvé $A=10, p=0,05$, on aura pour $K$ :

$$
100-100 \times \frac{1000 \times 0,05}{1000-10}=100-100 \times 0,0505
$$

$K=94,95 \%$ de propreté, au lieu de 95 si l'on ne tenait pas compte du mouillage.

On comprendra qu'une telle différence ne peut être retenue quand on songe qu'un mouillage à $10 \mathrm{~cm}^{3}$ ne l'affecte pas davantage, et que d'autre part, le dosage des impuretés ne peut être qu'approximatif. Aussi en pratique, l'expression $K=100-100$ p reste suffisante pour tous les cas où la détermination du coefficient de propreté peut avoir son utilité d'ordre hygiénique et psychologique.

Microscopie. - Elle complète la détermination quantitative des impuretés par l'étude de leurs qualités, ou pour mieux dire de leur nature et souvent de leur origine.

Pour des propretés ou malpropretés pondéralement égales, on pourra classer les laits. Un lait dont la majorité des corps étrangers sera composée de débris végétaux intestinaux ne vaudra évidemment pas, à poids égal, celui qui présentera des débris naturels n'ayant pas fait la traversée digestive.

On pourra consulter à cet égard le travail de LotH, dans la Revue Le Lait, de juin-juillet, août-septembre 1930.

Sur un coefficient d'altération du lait. - A propos de l'acidité du lait, $\mathrm{j}$ 'ai fait allusion plus haut à un coefficient d'altération qu'on pourrait obtenir en dosant l'acidité totale, ou niieux encore l'acide lactique total, libre ou combiné. Cette acidité est une variable de la fonction complexe du milieu lacté, variable par conséquent avec sa 
composition bactério-biophysico-chimique (d'origine sanguine et d'origine intra ou extra mammaire), dune en relation avec sa concentration moléculaire, les proportions relatives des différents composants : protides, lipides, glucides et lactose en particulier, avee la température, le temps écoulé, les ensemencements microbiens, les apports étrangers, solides, liquides et gazeux.

La détermination de cette variable peut done témoigner de la variation de la fonction dont elle est la conséquence. Elle peut être considérée comme l'expression des modifications, des altérations de l'équilibre vital du milieu vivant qu'est le lait à son origine sécrétoire.

Comme l'acidité lactique est normalement premier témoin de cette altération jusqu'à la coagulation du lait - moment limite où ce liquide perd nécessairement son nom - son évaluation peut présenter un certain intérêt, surtout si on le présente sous la forme d'un coefficient analogue au coefficient de propreté, et, de plus, mis en parallèle avec lui.

Mais ce nouveau coefficient, que j'appellerai coefficient d'altération, s'il dépend du coefficient de propreté, lequel entraîne tout d'abord et surtout la responsabilité du producteur, dépend encore de tant d'autres facteurs, la température en particulier, qu'on ne saurait, même à l'entrée de l'usine, l'imputer principalement au producteur.

Je ne lui accorde donc qu'un rôle d'appoint complémentaire au coefficient de propreté, mais rôle d'appoint toujours utile et même nécessaire quand le premier ne peut être déterminé. Pour le rendre comparable à ce dernier, on pourrait le calculer de la même manière, en prenant pour maximum d'acidité possible celle qui entraîne la coagulation avee le conoours de tous les facteurs favorables à cette coagulation (température, état météorique, etc.).

Soit donc $a$ l'acidité lactique totale (pour un litre) divisée par 10 , sans tenir compte de l'acidité au sortir de la mamelle, laquelle est à peu près constante à 1 gr. 50 par litre, sans tenir compte même de l'hyperlacticité ou de l'hypolacticité de ce liquide (l'une et l'autre dérivant de certaines maladies comme la tuberculose), ces nombres étant toujours peu élevés en face de l'acidité d'altération. On aura comme pour le coefficient de propreté, en désignant par $a$ les fractions de gramme de 0 à 1 :

$$
100 \mathrm{a}=\mathrm{K}^{\prime}
$$

Pour $\mathrm{a}=0$, le lait sera d'une acidité nulle.

Pour $\mathrm{a}=1$, le lait sera d'une acidité égale à $100 \%$.

Comme on le sait, on admet que 1 gramme d'acide lactique pour $100 \mathrm{~cm}^{3}$ ou 10 grammes pour 1000 ou 1 litre de lait représente un maximum compatible avec un lait non coagulé. 
$a$ variant de 0 à 1 donnera toutes les valeurs intermédiaires du coefficient d'altération K'.

Une étude comparative pourrait être faite entre les deux coefficients K (coefficient de propreté) et K'. Elle serait peut-être instructive.

Le coefficient de propreté du lait est le témoin causal ou étiologique. Le coefficient d'altération est le témoin conséquent ou symptomatique. Nous en avons également fait l'étude avec la dilution ou la concentration du lait.

\title{
L'EXACTITUDE DES DIFFÉRENTES MIÉTHODES DE CONTROLE LAITIER (1).
}

\author{
par Stefan TAUSSIG.
}

A la suite du Congrès international d'Agriculture de Prague et du Congrès mondial du Lait de Copenhague, et quelques semaines arant le récent Congrès mondial du Lait (Rome 1934) et le Congrès international d'Agriculiure (Budapest 1934), M. Stefan TAussig mettait en évidence : "Quelques-uns des aspeets de cette question, en utilicant les no breux travaux qui traitent des aptitudes et de la valeur des diverses méthodes de contrôle adoptées par les différents pays. "

En ce moment, les discussions ne sont pas terminées concernant : "Ies possibilités et l'opportunité d'une unification ou réglementation internationale du contrôle des vaches laitières, unification qui aurait pour but de rendre plus comparables les résultats du contrôle laitier dans les différents pays du monde $[35,37,38]$ (2). "

Aussi avons-nous cru utile de reproduire ici ce très intéressant travail de mise au point.

La Rédaction.

Les travaux dont il s'agit se basent en général sur des recherches ou des calculs faits à l'aide des données fournies par les sociétés de contrôle existantes. Ces recherches et ces calculs ont pris naissance avec les commencements mêmes du contrôle laitier (1878) et ont été depuis lors l'objet d'efforts de nombreux chercheurs dans le monde. Plus récemment, VoGEL, de Göttingen [16, 17], Warren Gifford [13], de l'Université de Missouri, ainsi que J. Houston et R. W. HaLe (Irlande du Nord) [39, 40], se sont occupés plus à fond de la question. Dans un de ses travaux, VogEL [17] passe en revue un grand nombre d'autres travaux plus anciens dans ce domaine et facilite ainsi beaucoup notre tâche d'une mise au point de ce problème.

Dans le contrôle laitier, tel qu'il est appliqué en pratique, il

(1) Revue internationale d'Agricul ture. Rome, XXVe année, No1, p. 3 T, janvier 1934.

(2) Las chiffres or tre crochets se réfèrent à la Bibliographie terminant cet article. 